\title{
La gestione dell'iperparatiroidismo secondario nella malattia renale cronica allo stadio 3 e 4
}

\author{
M. Cozzolino, L. Olivi, S. Pasho, G. Fallabrino, E. Volpi, P. Ciceri \\ U.O. Nefrologia e Dialisi, A.O. San Paolo, Cattedra di Nefrologia, Università di Milano, Milano
}

\section{Introduzione}

Liperparatiroidismo secondario è una delle complicanze più frequenti in corso di malattia renale cronica allo stadio 3 e 4 . L'IPTS è caratterizzato da aumentata sintesi e secrezione di PTH, iperplasia delle ghiandole paratiroidee e squilibrio a carico degli ioni bivalenti $(1,2)$. Esso è conseguenza di fattori quali l'ipocalcemia, la ritenzione di fosfati e la riduzione della sintesi di calcitriolo. In questi complessi meccanismi, altri fattori sono implicati nel determinare l'alterata sintesi e la secrezione di PTH, come il carico di alluminio, il diabete, gli estrogeni e le catecolamine (3). L’incremento dei livelli sierici di PTH è inoltre responsabile di una serie di conseguenze cliniche a lungo termine il cui target primario è lo scheletro. Oltre a ciò, si aggiunge una tossicità sistemica dell'IPTS a carico dell'apparato cardiovascolare, che spiega l'aumentato rischio di morbilità e mortalità cardiovascolare dei pazienti con CKD.

Nonostante gli intensi studi, i meccanismi che sono alla base dell'aumentata mortalità cardiovascolare dei pazienti uremici non sono noti, tuttavia si può assumere che l'imputato principale sia la deposizione di calcio a livello vascolare, che è stato identificato come un sicuro predittore di mortalità (5-7). Da tutto ciò si evince che il mantenimento dei livelli ottimali di PTH, fosforo e calcio sierici sia da considerare la base per scelte terapeutiche appropriate.

\section{Fisiopatologia}

Negli Stati Uniti circa il 38\% dei pazienti allo stadio 3 e il 68\% di quelli allo stadio 4 rientrano nei criteri diagnostici per l'IPTS. Molti studi dimostrano come, nella pratica clinica, né l'ipocalcemia né l'iperfosfatemia sono indicatori affidabili di IPTS. Al contrario, un metodo affidabile per l'individuazione di IPTS in fase precoce è la concentrazione di PTH sierico per dosaggi multipli una volta che la velocità di filtrazione glomerulare (eGFR) scenda al di sotto di 60 $\mathrm{mL} / \mathrm{min} / 1.73 \mathrm{~m}^{2}$ (Tab. I).

L'IPTS che si sviluppa in corso di CKD è determinato prevalentemente da tre fattori quali l'ipocalcemia, l'iperfosforemia e il deficit di $1,25(\mathrm{OH})_{2} \mathrm{D}_{3}$. Lo squilibrio nei livelli di fosforo e vitamina $\mathrm{D}$, in parte per via indiretta attraverso l'ipocalcemia, stimola le cellule paratiroidee a proliferare (12), inducendo iperplasia delle ghiandole paratiroidee con conseguente aumentata sintesi e secrezione di PTH (8-11). Inoltre calcio e $1,25(\mathrm{OH})_{2} \mathrm{D}_{3}$ regolano la trascrizione del gene del PTH riducendo i propri recettori presenti sulle ghiandole paratiroidee, riduzione che le rende resistenti alla soppressione della secrezione di PTH da parte di calcio e $1,25(\mathrm{OH})_{2} \mathrm{D}_{3}$ (4) (Fig. 1).

Gli alti livelli di PTH inducono la sindrome tipica dell'IPTS caratterizzata da disturbi a livello cardiovascolare (ipertrofia ventricolare sinistra e insufficienza cardiaca congestizia) e osseo, causando un aumento 
Fig. 1 - Effetto della riduzione della massa renale nella malattia renale cronica.

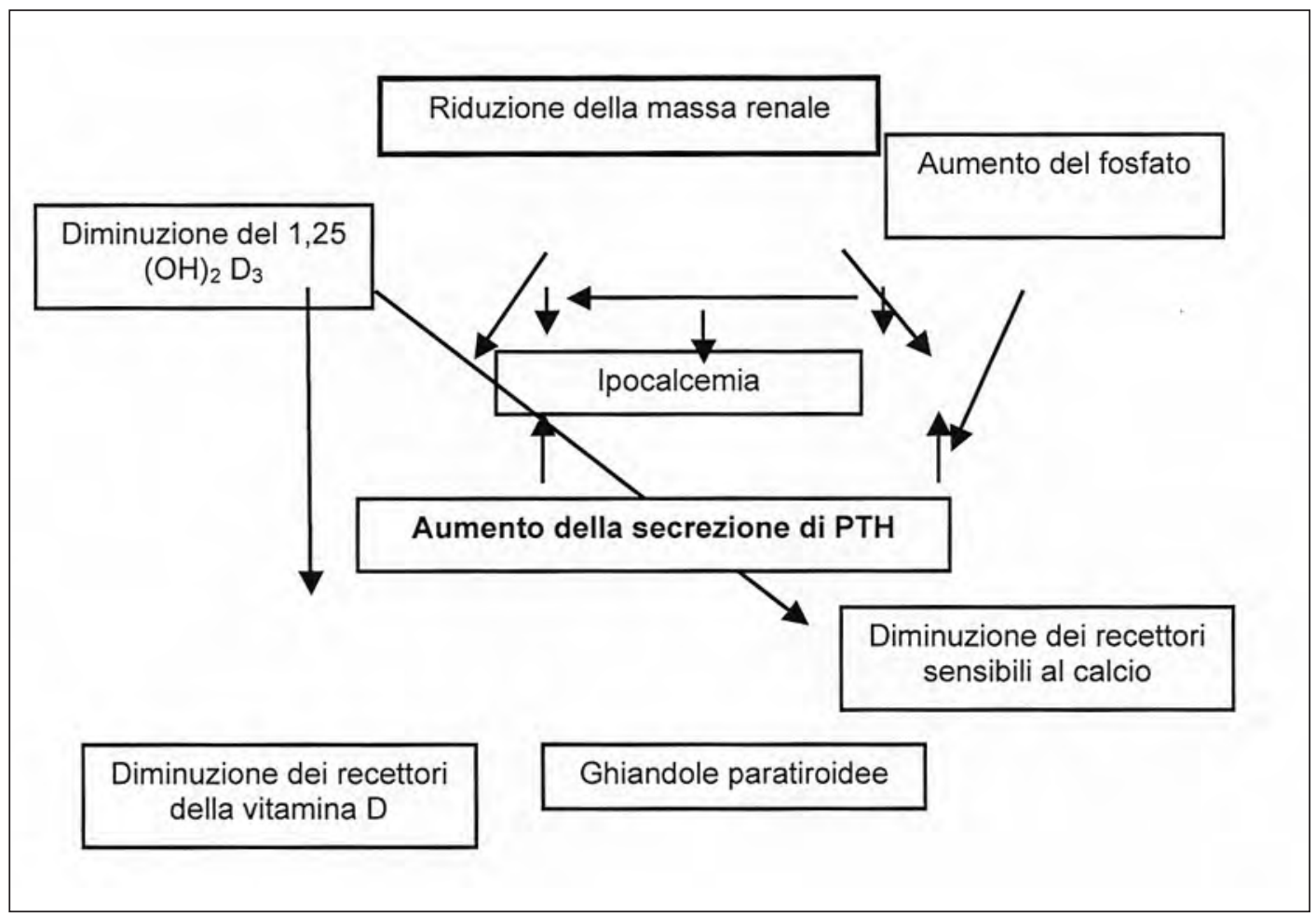

TABELLA I - I LIVELLI TARGET RACCOMDANDATI ATTUALMENTE NELLA MALATTIA RENALE CRONICA ALLO STADIO 3 E 4

\begin{tabular}{ll}
\hline Parametri & Raccomandazioni \\
\hline PTH intatto $(\mathrm{pg} / \mathrm{mL})$ & $\begin{array}{l}\text { CKD stadio } 3 \\
\text { (GFR stimata } 30-59 \mathrm{~mL} / \mathrm{min}): 35-70\end{array}$ \\
& CKD stadio 4 \\
& (GFR stimata $15-29 \mathrm{~nL} / \mathrm{min}): 70-110$
\end{tabular}

$\mathrm{PTH}=$ paratormone; $\mathrm{CKD}=$ chronic kidney disease $; \mathrm{GFR}=$ glomerular filtration rate; $\mathrm{Ca}=$ calcio; $\mathrm{P}=$ fosforo

della mortalità (13-15). Negli ultimi anni, vista l'influenza reciproca fra la patologia cardiovascolare e ossea nell'IPTS, si tende a utilizzare un unico termine che le definisce entrambe: "bone-vascular disease".

\section{Il trattamento nelle fasi 3 e 4 della malattia renale cronica: le basi teoriche per un intervento precoce}

È noto che quando decresce il filtrato glomerulare (GFR), si riduce anche l'1-alfa idrossilasi renale e di conseguenza diminuiscono i livelli sierici di 1,25-diidrossivitamina D. Questo evento si osserva piuttosto precocemente, a partire dallo stadio 2 della CKD (GFR
$60-90 \mathrm{~mL} / \mathrm{min}$ ), quando ancora non si sono verificate variazioni dei livelli di fosforemia e calcemia (16). A mano a mano che l'insufficienza renale progredisce, aumenta il numero di pazienti con bassi livelli di calcitriolo circolanti ed elevati livelli di PTH. Il deficit di calcitriolo innesca 2 eventi principali: 1) la riduzione dell'assorbimento intestinale di calcio con conseguente ipocalcemia e 2) un ridotto controllo genomico sul pre-pro-PTHmRNA; entrambi gli eventi rappresentano uno stimolo importante alla sintesi e secrezione del PTH. Inoltre, negli stadi 3 e 4 della CKD esiste la peggiore combinazione di eventi poiché gli aumentati livelli di fosforo sierici possono essere un ulteriore stimolo per la secrezione di PTH, indipendentemente dall'effetto dei bassi livelli di vitamina D.

In questa condizione l'acidosi metabolica (17) è inoltre capace di ridurre ulteriormente l'attività dell'1-alfaidrossilasi e di rendere l'osso più sensibile nei confronti del PTH. Un'altra condizione che può essere alla base dell'aumentata sintesi di PTH nei pazienti uremici è la carenza di 25OH-idrossivitamina D (25OHD), che può essere presente anche in pazienti con funzione renale normale (18). In questi casi in teoria si apre la possibilità di usare il 25OHD nella terapia di un particolare sottogruppo di pazienti uremici affetti da iperparatiroidismo secondario. Tuttavia, Gallieni et al (19) hanno dimostrato che il calcitriolo è essenziale nel potenziare l'uptake del 250HD all'interno dei monociti, 
suggerendo quindi che entrambi, il $250 \mathrm{OHD}$ e il calcitriolo, devono essere dati in combinazione nei casi di pazienti uremici con deficit di 25OHD. Questa affermazione si basa anche sull'osservazione che il calcitriolo potenzia l'espressione della megalina a livello tubulare (20), essenziale per il passaggio del 25OHD ultrafiltrato all'1-alfa-idrossilasi nel tubulo renale prossimale.

Molti studi hanno recentemente dimostrato che i pazienti affetti da insufficienza renale cronica, anche nelle fasi precoci della loro malattia, possono già presentare calcificazioni vascolari e quindi essere soggetti a coronaropatie, vasculopatia periferica, ipertrofia cardiaca e outcome infausti (21-25). Il carico di fosforo è considerato il maggiore responsabile delle calcificazioni vascolari attraverso la stimolazione del Core binding factor alpha 1 (Cbfa 1), un fattore in grado di mutare il fenotipo delle cellule muscolari lisce vascolari in cellule simil-osteoblastiche (26).

L'acquisizione di un'adeguata strategia e del giusto timing per il trattamento dell'iperparatiroidismo secondario nei pazienti con CKD diventa di cruciale importanza. La valutazione preliminare dei livelli sierici della 25-idrossivitamina D e l'eventuale conseguente loro correzione mediante l'utilizzo dell'ergocalciferolo per ridurre i livelli di PTH e promuovere il fronte di mineralizzazione osseo e la deposizione di calcio nelle ossa, godono di un consenso generale (28). Mentre non esiste alcuno studio che indichi una maggiore appropriatezza circa l'uso del $25 \mathrm{OHD}_{3}$ nei pazienti con CKD, parecchi studi mostrano l'effetto positivo del calcitriolo, del doxecalciferolo e del paracalcitolo sulla soppressione del PTH in pazienti con CKD moderata $(29,30)$. La supplementazione con calcitriolo in fase precoce nei pazienti con CKD trova supporto in recenti evidenze che indicano che in fase 3 e 4 la prevalenza dei pazienti con bassi livelli di calcitriolo ed elevato PTH è alta (Tab. II) (16).

È noto che l'osteopenia e l'osteoporosi sono fattori prognostici negativi per quel che riguarda la sopravvivenza dei pazienti con insufficienza renale cronica. Perciò diventa necessario adottare qualsiasi tipo di strategia mirata ad incrementare la mineralizzazione ossea per migliorare gli outcome scheletrici e cardiovascolari. I possibili effetti positivi della somministrazione precoce degli analoghi della vitamina $\mathrm{D}$ ai pazienti in fase $3 \mathrm{e}$ 4 della CKD sono supportati da dati recentemente pubblicati da Rix et al (31), che dimostrano come l'alfacalcidiolo somministrato per parecchi mesi sia in grado di ridurre il turnover osseo e di aumentare la densità minerale ossea, nonché di ottenere un soddisfacente controllo del PTH. Tuttavia ancora mancano studi a lungo termine sugli effetti del paracalcitolo e del doxecalciferolo sulla densità minerale ossea, sull'istologia ossea $\mathrm{e}$ sulle fratture.

Comunque sia, considerato che anche modeste elevazioni dei valori di PTH (per esempio $>60 \mathrm{pg} / \mathrm{mL}$ ) sono associate con una significativa perdita ossea nei pazienti in fase 3 e 4 della CKD, è consigliabile mantenere i livelli di PTH all'interno del normale range non solo per prevenire la perdita ossea e l'iperplasia paratiroidea, ma anche per controllare i possibili effetti negativi del deficit di vitamina $\mathrm{D}$ sull'apparato cardiovascolare.

\section{Come trattare l'iperfosforemia nella CKD allo stadio 3 e 4}

La dieta si è spesso rivelata insufficiente per il controllo dell'iperfosfatemia, nonostante ciò può essere considerata come un coadiuvante della terapia farmacologica. Si raccomanda ai pazienti di non ingerire cibi ad alto contenuto di fosforo, ponendo particolare attenzione a non modificare eccessivamente la dieta inducendo malnutrizione.

La raccomandazione delle linee guida $\mathrm{K} / \mathrm{DOQI}$ e quella di mantenere il fosforo sierico tra 2.7 e $4.6 \mathrm{mg} / \mathrm{dL}$ (32).

\section{Il calcio carbonato come chelante in CKD allo stadio 3 e 4 ?}

Il chelante maggiormente utilizzato in clinica fino a ora per il controllo dell'iperfosforemia è il calcio carbona-

TABELLA II - PREVALENZA DEI PAZIENTI AFFETTI DA CKD IN FASE 3 E 4 CON RIDOTTI LIVELLI SIERICI DI CALCITRIOLO (<35 PG/ML) O ELEVATI LIVELLI SIERICI DI PTH (>65 PG/ML) (DA LEVIN ET AL, 2005)

\begin{tabular}{lccc}
\hline Stadio CKD & $\begin{array}{c}\text { \% pz con ridotti livelli } \\
\text { sierici di calcitriolo }\end{array}$ & $\begin{array}{c}\text { \% pz con elevati livelli } \\
\text { sierici di PTH }\end{array}$ & N \\
\hline 3 & 61 & 38 & 1.109 \\
4 & 87 & 68 & 262 \\
\hline
\end{tabular}


to, le cui dosi vengono aumentate gradualmente fino a raggiungere il target di fosforo sierico desiderato. Il principale problema correlato con l'utilizzo dei sali di calcio è che l'assorbimento gastrointestinale di parte del calcio somministrato può favorire lo sviluppo di calcificazioni coronariche, per tale motivo le $\mathrm{K} / \mathrm{DOQI}$ suggeriscono che la dose totale di calcio elementare assunta giornalmente non debba superare i $2000 \mathrm{mg}$ (32).

\section{Il Sevelamer HCl o il Sevelamer Carbonato in CKD allo stadio 3 e 4?}

Sevelamer è stato il primo chelante del fosforo privo di alluminio e di calcio in grado di ridurre i livelli di fosforo senza causare ipercalcemia, mantenendo al tempo stesso la capacità di sopprimere la produzione di PTH. Sevelamer rallenta infatti la progressione delle calcificazioni vascolari nei pazienti in emodialisi portando così ad una riduzione della mortalità (33). Un ulteriore vantaggio di questo farmaco è la sua capacità di ridurre i livelli di colesterolo LDL e totale. Il principale effetto collaterale è che la somministrazione a lungo termine riduce il bicarbonato sierico in maniera superiore ai sali di calcio.

\section{Il lantanio carbonato in CKD allo stadio 3 e 4?}

Il lantanio e un metallo recentemente approvato negli USA per il trattamento dell'iperfosforemia nei pazienti in dialisi. Si è dimostrato efficace, ben tollerato e gli eventi avversi più frequenti sono di tipo gastrointestinale (nausea e vomito). L'uso diffuso di tale farmaco può essere limitato dal timore della tossicità associata ad una esposizione a lungo termine, essendo sconosciuti gli effetti di un eventuale accumulo. Recentemente si sta valutando l'ipotesi di un suo utilizzo nel controllo dei livelli di fosforo in predialisi vista la sua efficacia e tollerabilità (34).

\section{VDRAs (Vitamin D Receptor Activators)}

La sintesi di PTH e la proliferazione delle cellule paratiroidee è controllata e subordinata all'attivazione dei recettori della vitamina D (VDR) (8). Ciò diventa evidente in caso di insufficienza renale quando, a causa dei bassi livelli di vitamina $\mathrm{D}$ circolante, si ha diminuzione del numero di VDR a livello paratiroideo con conseguente aumento della sintesi di PTH e iperplasia paratiroidea (35). Dato il meccanismo d'azione della vitamina $\mathrm{D}$, sono stati sviluppati dei farmaci con azione di attivazione dei VDR quali ad esempio calcitriolo e paracalcitolo, che inibendo specifici fattori di crescita sono in grado di controllare la proliferazione delle cellule paratiroidee. I farmaci di questa classe si dividono in tre classi con calcitriolo che appartiene alla prima generazione e paracalcitolo alla terza. Il paracalcitolo è il farmaco più interessante di questa classe per la riduzione dell'effetto iperfosforemico e ipercalcemizzante presenti nel calcitriolo, probabilmente dovuto alla diminuzione dell'assorbimento di calcio e fosforo a livello intestinale (36). L'utilità degli attivatori dei VDR è dimostrata in studi retrospettivi dove viene dimostrato che il trattamento con questi farmaci riduce la mortalità per tutte le cause (37) e migliora la morbidità (38). Il paracalcitolo ha una finestra terapeutica più ampia dei suoi predecessori e il suo utilizzo nei pazienti con CKD in fase 3 e 4 ha il suo razionale nell'efficacia di questo farmaco nella soppressione dei livelli di PTH con minimo effetto sui livelli di calcio e fosforo.

\section{Il Cinacalcet in CKD allo stadio 3 e 4}

L'attuale terapia dell'IPTS del paziente uremico ha un successo limitato nell'ottenere il raggiungimento dei valori di PTH, calcio e fosforo indicati dalle linee guida internazionali (NKF-K/DOQI) (32). I calciomimetici, composti in grado di aumentare la sensibilità del sensore del calcio (CaSR), interagendo con il dominio intramembranoso del CaSR stesso, si sono dimostrati nelle esperienze precliniche efficaci nel ridurre i livelli di PTH, impedendo lo sviluppo dell'iperplasia ghiandolare, inducendo la regressione dell'osteite fibrosa e prevenendo lo sviluppo delle calcificazioni vascolari (39). Gli studi clinici che hanno esclusivamente utilizzato il Cinacalcet $\mathrm{HCl}$, hanno confermato l'efficacia del farmaco sia nel controllo dell'IPTS nel paziente in dialisi che, più recentemente, nei soggetti affetti da iperparatiroidismo primitivo. In particolare, nel soggetto uremico in dialisi, il Cinacalcet ha dimostrato un profilo di efficacia importante, riducendo i livelli di PTH senza aumentare quelli di calcio e di fosforo (40). Gli effetti collaterali riportati sono riferibili a disturbi gastroenterici e ipocalcemia, il più delle volte asintomatica (41).

L'uso del Cinacalcet è stato inoltre prospettato nel controllo dell'IPTS persistente dopo il trapianto di rene e nei soggetti con CKD allo stadio 3-4 (42). Prime esperienze sembrano incoraggianti in tal senso. Il limite più importante della terapia affidata ai calciomimetici è che essa non costituisce una correzione patogenetica della patologia osteo-metabolica dell'IPTS e deve pertanto essere affiancata alle terapie che agiscono in modo correttivo su quelli che sono gli elementi ezio- 
patogenetici certi (deficit e resistenza alla vitamina $\mathrm{D}$, bilancio positivo del fosforo, bilancio negativo calcico). L'uso dei calciomimetici, che rappresenta comunque un importante avanzamento nel campo della terapia dell'IPTS dell'uremia, dovrà inoltre essere oggetto di uno stretto controllo clinico per le potenziali interferenze con altri sistemi cellulari dove le sue funzioni sono ancora poco note.

\section{Riassunto}

Una delle complicanze più frequenti in corso di malattia renale cronica $(\mathrm{CKD}=$ Chronic Kidney Disease $)$ allo stadio 3 e 4 è rappresentata dall'iperparatiroidismo secondario (IPTS). Le calcificazioni e la patologia cardiovascolare sono spesso presenti nei soggetti affetti da CKD e rappresentano la prima causa di mortalità nei pazienti in dialisi, cosicché la prevenzione e la terapia dell'IPTS e delle alterazioni del metabolismo calcio-fosforico rappresentano due passaggi chiave nel trattamento dei pazienti con CKD non ancora in dialisi. Il trattamento classico dell'IPTS, nei pazienti affetti da
$\mathrm{CKD}$, con calcitriolo e chelanti del fosforo a base di sali di calcio ha portato ad aumento della deposizione di calcio-fosfato nei tessuti molli. Per i pazienti in dialisi, studi recenti hanno documentato come i nuovi chelanti del fosforo non contenenti né calcio né alluminio (sevelamer, lantanio carbonato), gli attivatori selettivi del recettore della vitamina $D$, VDRAs (paracalcitolo), meno ipercalcemizzanti e iperfosforemizzanti, e i calciomimetici (cinacalcet) siano efficaci nel trattamento dell'IPTS e potenzialmente utili nella prevenzione dei processi di calcificazione extrascheletrica.

Pertanto, i calciomimetici e i VDRAs rappresentano una doppia arma terapeutica per ridurre il rischio di patologia cardio-vascolare nei pazienti con CKD affetti da IPTS.

Indirizzo degli Autori:

Mario Cozzolino, $\mathrm{MD}, \mathrm{PhD}$

U.O. Nefrologia e Dialisi, AO San Paolo

Cattedra di Nefrologia, Università di Milano

Via A. di Rudini, 8

20142 Milano, Italy

mariocozzolino@hotmail.com

\section{Bibliografia}

1. Silver J. Molecular mechanisms of secondary hyperparathyroidism. Nephrol Dial Transplant 2000; 15 (Suppl 5): S2-7.

2. Slatopolsky E, Brown A, Dusso A. Pathogenesis of secondary hyperparathyroidism. Kidney Int 1999; 56 (Suppl 73): S14-9.

3. Bro S, Olgaard K. Effects of excess PTH on nonclassical target organs. Am J Kidney Dis 1997; 30: 606-20.

4. Brown EM, Gamba G, Riccardi D, et al. Cloning and Charaterization of an extracellular Ca2-sensing receptor from bovine parathyroid. Nature 1993; 366: 575-80.

5. Blacher J, Guerin AP, Pannier B, Marchais SJ, London GM. Arterial calcifications, arterial stiffness, and cardiovascular risk in end-stage renal. Hypertension 2001; 38: 938-42.

6. Slagueira M. Vascular calcification in the uremic patient: a cardiovascular risk? Kidney Int 2003; 63 (Suppl): S119-21.

7. Wang AY, Wang M, Woo J, et al. Cardiac valve calcification as an important predictor for all-cause mortality and cardiovascular mortality in long-term peritoneal dialysis patients: a prospective study. J Am Soc Nephrol 2003; 14: 159-68.
8. Parfitt AM. The hyperparathyroidism of chronic renal failure: a disorder of growth. Kidney Int 1997; 52: 3-9.

9. Cozzolino M, Butti A, Chiarelli G, et al. New insights in the pathogenesis of secondary hyperparathyroidism. G Ital Nefrol 2005; 4: 329-36.

10. Slatopolsky E, Finch J, Denda M, et al. Phosphorus restriction prevents parathyroid gland growth. High Phosphorus directly stimulates PTH secretion in vitro. J Clin Invest 1996; 97: 2534-40.

11. Silver J, Bar Sela S, and Naveh-Many T. Regulation of parathyroid cell proliferation. Curr Opin Nephrol Hypertens 1997; 6: 321-26.

12. Cozzolino M, Brancaccio D, Gallieni M, et al. Pathogenesis of parathyroid hyperplasia in renal failure. J Nephrol 2005, 18 (1): 5-8.

13. Cozzolino M, Dusso A, Slatopolsky E. Role of calcium x phosphate product and bone associated proteins on vascular calcification in renal failure. J Am Soc Nephrol 2001; 12: 2511-6.

14. Cozzolino M, Brancaccio D, Gallieni M, Slatopolsky E. Pathogenesis of vascular calcification in chronic kidney disease. Kidney Int 2005; 68: 429-36.

15. Cozzolino M, Gallieni M, Brancaccio D, et al. Vitamin D retains an important role in the pathogenesis and management of secondary hyperparathyroidism in chronic 
renal failure. J Nephrol 2006; 19: 566-57.

16 Levin A, Andress LD, Molitch M, et al. Prevalence of abnormal PTH in relation to serum calcium, phosphorus and vitamin $\mathrm{D}$ in patients with stage 3 and 4 Chronic Kidney Disease (CKD). JASN 2005 (Abstract Meeting ASN 2005).

17. Kawashima H, Kraut JA, Kurokawa K. Metabolic acidosis suppresses 25-hydroxyvitamin D3-1alpha-hydroxylase in the rat kidney. J Clin Invest 1982; 70: 135-40.

18. Nesby-O'Dell S, Scanlon KS, Cogswell ME, et al. Hypovitaminosis $\mathrm{D}$ prevalence and determinants among African American and white women of reproductive age: Third National Health and Nutrition Examination Survey, 1988-1994. Am J Clin Nutr 2002; 76: 187-92.

19. Gallieni M, Kamimura S, Ahmed A, et al. Kinetics of monocyte $1 \alpha$-hydroxylase in renal failure. Am J Physiol 1995; 268: F746-53.

20. Liu W, Yu WR, Carling T, et al. Regulation of gp330/ megalin expression by vitamins A and D. Eur J Clin Invest 1998; 28: 100-07.

21. Raggi P, Boulay A, Chasin-Taber S, et al. Cardiac calcification in adult hemodialysis patients. A link between end-stage renal disease and cardiovascular disease? J Am Coll Cardiol 2002; 39: 695-701.

22. London GM, Guerin AP, Marchais SJ, et al. Arterial medial calcification in end-stage renal disease: impact on all cause and cardiovascular mortality. Nephrol Dial Transplant 2003; 18: 1731-40.

23. Yildiz A, Memisoglu E, Oflaz $\mathrm{H}$, et al. Atherosclerosis and vascular calcification are independent predictors of left ventricular hypertrophy in chronic hemodialysis patients. Nephrol Dial Transplant 2005; 20: 760-7.

24. Guerin AP, Blacher J, Pannier B, et al. Impact of aortic stiffness attenuation on survival of patients in end-stage renal failure. Circulation 2001; 103: 987-92.

25. Blacher J, Guerin AP, Pannier B, et al. Arterial calcifications, arterial stiffness and cardiovascular risk in endstage renal disease. Hypertension 2001; 38: 938-42.

26. Ducy P, Zhang R, Geoffroy V, et al. Osf2/Cbfa1: a transcriptional activator of osteoblast differentiation. Cell 1997; 89: 747-54.

27. Sjoden G, Lindgren JU, DeLuca HF. Antirachitic activity of 1 alpha-hydroxyergocholecalciferol and 1 alpha-hydroxycholecalciferol in rats. J Nutr 1984; 114: 2043-6.

28. Gonzalez EA, Sachdeva A, Oliver DA, Martin KJ. Vitamin D insufficiency and deficiency in chronic kidney disease: a single center observational study. Am J Nephrol 2004; 24: 503-10.

29. Healy MD, Malluche HH, Goldstein DA, et al. Effects of long-term therapy with calcitriol in patients with moderate renal failure. Arch Intern Med 1980; 140: 1030-33.

30. Coburn JW, Maung HM, Elangovan L, et al. Doxercalciferol safely suppresses PTH levels in patients with secondary hyperparathyroidism associated with chronic kidney disease stages 3 and 4. Am J Kidney Dis 2004; 43: 877-90.

31. Rix M, Andreassen H, Eskildsen P, et al. Bone mineral density and biochemical markers of bone turnover in patients with pre-dialysis chronic renal failure. Kidney Int 1999; 56: 1084-93.

32. National Kidney Foundation. K/DOQI clinical practice guidelines for bone metabolism and disease in chronic kidney disease. Am J Kidney Dis 2003; 42 (Suppl 3): S1201.

33. Caglar K, Yilmaz MI, Saglam M, et al. Short-term treatment with sevelamer increases serum fetuin-a concentration and improves endothelial dysfunction in chronic kidney disease stage 4 patients. Clin J Am Soc Nephrol 2008; 3: 61-8.

34. Sprague SM, Abboud H, Qiu P, et al. Lanthanum carbonate reduces phosphorus burden in patients with CKD stages 3 and 4: a randomized trial. Clin J Am Soc Nephrol. 2009; 4: 178-85.

35. Szabo A, Merke J, Beier E, et al. 1,25(OH)2vitamin D3 inhibits parathyroid cell proliferation in experimental uremia. Kidney Int 1989; 35: 1049-56.

36. Slatopolsky E, Cozzolino M, Finch JL. Differential effects of 19-nor-1,25- $(\mathrm{OH}) 2 \mathrm{D} 2$ and 1alpha-hydroxyvitamin D2 on calcium and phosphorum in normal and uremic rats. Kidney Int 2002; 62:1277-84.

37. Teng M, Wolf M, Ofsthun MN, et al. Activated injectable vitamin $\mathrm{D}$ and hemodialysis survival: a historical cohort study. J Am Soc Nephrol 2005; 16: 1115-25.

38. Dobrez DG, Mathes A, Amdahl M, et al. Paricalcitoltreated patients experience improved hospitalization outcomes compared with calcitriol-treated patients in real-world clinical settings. Nephrol Dial Transplant 2004; 19: 1174-81.

39. Kawata T, Imanishi Y, Kobayashi K, el al. Relationship between parathyroid calcium sensing receptor expression and potency of the calcimimetic, cinacalcet, in suppressing parathyroid hormone secretion in an in vivo murine model of primary hyperparathyroidism. Eur J Endocrinol 2005; 153: 587-94.

40. Block G, Kevin JM, De Francisco ALM, et al. Cinacalcet for secondary hyperparathyroidism in patients receiving hemodialysis. N Engl J Med 2004; 350: 627-35.

41. Moe SM, Chertow GM, Coburn JW, et al. Achieving NFK-K/DOQI TM bone metabolism and disease treatment goals with cinacalcet $\mathrm{HCl}$. Kidney Int 2005; 67: 760-71.

42. Chonchol M, Locatelli F, Abboud HE, et al. A randomized, double-blind, placebo-controlled study to assess the efficacy an safety of cinacalcet $\mathrm{HCl}$ in participants with CKD not receiving dialysis. Am J Kidney Dis 2008; Dec 23. 